\title{
Hidden in plain sight: UVIT and MUSE discovery of a large, diffuse star-forming galaxy
}

\author{
Jyoti Yadav ${ }^{1,2} \odot$, Mousumi Das ${ }^{1}$, Sudhanshu Barway ${ }^{1} \odot$, and Francoise Combes $^{3} \odot$ \\ ${ }^{1}$ Indian Institute of Astrophysics, Koramangala II Block, Bangalore 560034, India \\ e-mail: jyoti@iiap.res.in \\ 2 Pondicherry University, R.V. Nagar, Kalapet 605014, Puducherry, India \\ 3 Observatoire de Paris, LERMA, College de France, CNRS, PSL University, Sorbonne University, 75014 Paris, France
}

Received 19 October 2021 / Accepted 22 December 2021

\begin{abstract}
We report the discovery of a nearby large, diffuse galaxy that shows star formation using Ultra Violet Imaging Telescope (UVIT) farUV observations and archival optical data from Multi-Unit Spectroscopic Explorer (MUSE), Dark Energy Camera Legacy Survey, and InfraRed Survey Facility near-infrared observations. The galaxy was not detected earlier due to its superposition with the background galaxy NGC 6902A. They were together mistakenly classified as an interacting system. NGC 6902A is at a redshift of 0.05554 , but MUSE observations indicate that the interacting tail is a separate star-forming foreground galaxy at a redshift of 0.00980 . We refer to the new galaxy as UVIT J202258.73-441623.8 (UVIT J2022). The near-infrared observations show that UVIT J2022 has a stellar mass of $8.7 \times 10^{8} M_{\odot}$. Its inner disk $(R<4 \mathrm{kpc})$ shows UV and $\mathrm{H} \alpha$ emission from ongoing massive star formation. The rest of the disk is extremely low in luminosity, has a low stellar surface density, and extends out to a radius of $R \sim 9 \mathrm{kpc}$. The velocity and metallicity distribution maps and the star formation history indicate that UVIT J2022 has undergone three bursts of star formation. The latest episode is ongoing, which is supported by the presence of widespread $\mathrm{H} \alpha$ and UV emission in its inner disk. The galaxy also shows patchy spiral arms in the far UV, and there is a metallicity enhancement along a bar-like feature. UVIT J2022 is thus a unique example of triggered star formation in a diffuse galaxy resulting in the growth of its inner stellar disk. Our study raises the intriguing possibilities that (i) there could be similar diffuse galaxies that have been mistakenly interpreted as interacting galaxies due to their superposition, and (ii) UV or $\mathrm{H} \alpha$ emission could be a way to detect such diffuse galaxies in our local universe.
\end{abstract}

Key words. galaxies: interactions - galaxies: star formation - techniques: imaging spectroscopy galaxies: individual: J202258.73-441623.8 - galaxies: individual: NGC 6902A

\section{Introduction}

Low surface brightness galaxies (LSBGs) are defined as galaxies with very diffuse stellar disks that have central surface brightnesses in the $B$ band $\left(\mu_{B}\right)$ fainter than $23 \mathrm{mag} \mathrm{arcsec}^{-2}$ (Impey \& Bothun 1997). However, in the past decade, several deep optical surveys have detected LSBGs down to much lower faintness levels of $26 \mathrm{mag} \operatorname{arcsec}^{-2}$ in the $r$ band (Venhola et al. 2018; Lim et al. 2020). The extremely faint and extended LSBGs are called ultra-diffuse galaxies (UDGs). Although the criteria vary in the literature, in general, UDGs have a central $g$ band $\left(\mu_{g}(0)\right)$ surface brightness fainter than $24 \mathrm{mag} \mathrm{arcsec}^{-2}$ and an effective radius larger than $1.5 \mathrm{kpc}$ (van Dokkum et al. 2015). They are classified as either blue (gas-rich) or red (gas-poor) UDGs. The gas-rich UDGs are present in the outskirts of galaxy groups. In contrast, the gas-poor UDGs are found in clusters, which suggests that the environmental processes are dominating (Leisman et al. 2017; Prole et al. 2019). These galaxies make up the tail end of the luminosity function (Koda et al. 2015) and may extend down to much lower levels than we would expect (Fattahi et al. 2020). The common unifying factor for all LSBGs and UDGs is that they have diffuse stellar disks, that is, their disks appear to have low stellar surface densities
(Sales et al. 2020), which can be connected to low star formation rates (Rong et al. 2020). Although LSBGs are historically considered to be gas-rich (Honey et al. 2018), UDGs may or may not contain gas (Leisman et al. 2017; Chowdhury 2019).

Another class of galaxies that have very low stellar density disks are the extended ultraviolet (XUV) disk galaxies, which show star formation in their extreme outer disks, where both stellar densities and metallicities are very low (Yadav et al. 2021a). The star formation in these adverse environments may have been triggered by gas inflow from nearby galaxies or cold gas accretion from the cosmic web (Sancisi et al. 2008). Taking this as an example, it is possible that UV or $\mathrm{H} \alpha$ emission from compact star-forming complexes (SFCs) in diffuse galaxies could be one of the ways to detect LSBGs or blue UDGs that may otherwise go unnoticed in larger surveys. Ultraviolet emission is especially important because it traces young $\mathrm{O}$, B stars for $10^{8}$ years, whereas $\mathrm{H} \alpha$ emission is produced by photoionization from massive stars $\left(M>10 M_{\odot}\right)$ and is sensitive to star formation for only $10^{6}$ to $10^{7}$ years (Das et al. 2021). Star formation is, however, difficult in low-density disks as the disk selfgravity is low. Therefore, triggers such as galaxy interactions and flybys are important, as shown in semi-analytical simulations (Somerville et al. 2001; Menci et al. 2005). This Letter reports the serendipitous discovery of a nearby diffuse galaxy that shows 

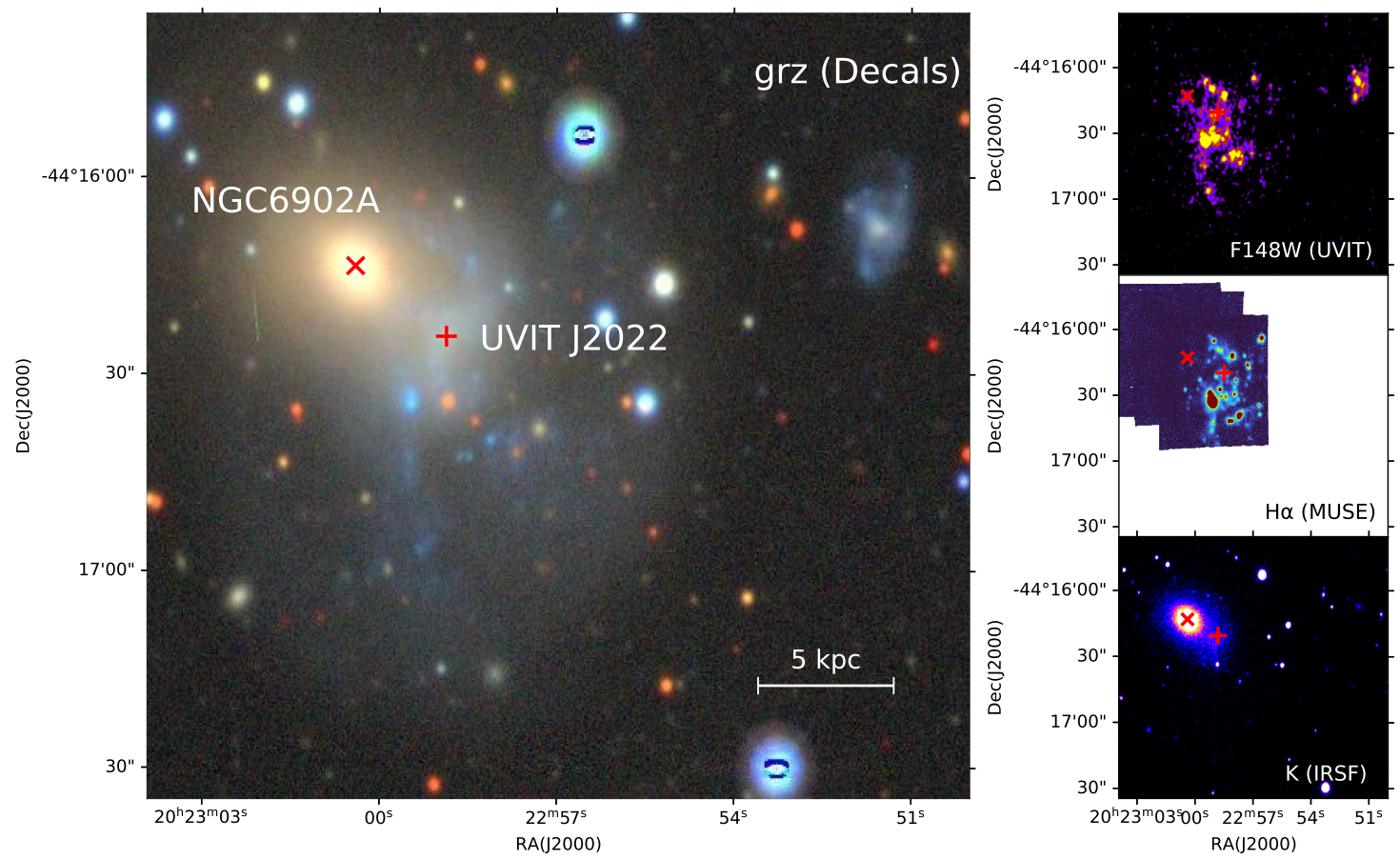

Fig. 1. Multi-wavelength images of UVIT J2022. The $g r z$ image shows the background galaxy, NGC 6902A, and the foreground galaxy, UVIT J2022. Star-forming regions in UVIT J2022 are prominent in FUV and $\mathrm{H} \alpha$. The $K$ band image reveals the emission from old stars in NGC 6902A. The + and $\times$ symbols in red indicate the kinematic centre of UVIT J2022 and NGC 6902A, respectively.

intense star formation in its inner disk using Ultra Violet Imaging Telescope (UVIT) and Multi-Unit Spectroscopic Explorer (MUSE) data. It is part of a larger study of star formation in a sample of southern interacting galaxies. This new galaxy lies in the foreground of NGC 6902A, which, as shown in this Letter, had been mistakenly classified as an interacting galaxy. The following sections describe our results and their implications for detecting LSBGs in our nearby universe. We have used flat cosmology with $\Omega_{\Lambda}=0.7, \Omega_{\mathrm{M}}=0.3$, and $H_{0}=70 \mathrm{~km} \mathrm{~s}^{-1} \mathrm{Mpc}^{-1}$.

\section{Observations}

\subsection{Imaging data}

We observed NGC 6902A using the UVIT on board Astrosat (Kumar et al. 2012). The UVIT has two telescopes, one for the far ultraviolet (FUV; 1300-1800 ) and another for the near ultraviolet (NUV; 2000-3000 ). The UVIT has a field of view of $28^{\prime}$ and spatial resolution of $\sim 1^{\prime \prime}$. The FUV observations were done in the $F 148 W$ band (1231-1731 $\AA$ ). We reduced the UVIT level 1 data using CCDLAB (Postma \& Leahy 2017, 2020).

We used archival $g, r$, and $z$ band data from the Dark Energy Camera Legacy Survey (DECaLS). DECaLS uses the Dark Energy Camera (DECam; Flaugher et al. 2015) mounted on the Victor M. Blanco telescope at the Cerro Tololo InterAmerican Observatory (CTIO). DECam has 62 charge-coupled devices with a $2048 \times 4096$ pixel format each for imaging. It has a field of view of $2.2^{\circ}$ in diameter and a pixel scale of 0.262 arcsec pixel $^{-1}$. DECam can achieve $5 \sigma$ depth in total exposure times of 166, 134, and $200 \mathrm{~s}$ in the $g, r$, and $z$ bands for an emission line galaxy with a half-light radius of $0.45^{\prime \prime}$.

The near-infrared observations of NGC 6902A were carried out using the SIRIUS camera (Nagayama et al. 2003) on the InfraRed Survey Facility (IRSF) $1.4 \mathrm{~m}$ telescope at South African Astronomical Observatory (SAAO) in Sutherland, South
Africa. The SIRIUS camera performs simultaneous imaging in the $J H K$ bands. It has a field of view of $7.7^{\prime} \times 7.7^{\prime}$ (Nagayama 2012). The exposure time of the images is $\sim 120 \mathrm{~min}$, and they were taken in automatic dithering mode with $\sim 20^{\prime \prime}$ steps with individual frame exposure times of 30 seconds each. We used the pipeline available for the SIRIUS observations to reduce the data, including corrections for non-linearity, dark subtraction, and flat fielding.

\subsection{Integral field unit data}

We used MUSE archival data (Bacon et al. 2010). MUSE gives $3 \mathrm{D}$ spectroscopic data cubes with high resolution. We used the data from the wide-field mode, which has a field of view of $1^{\prime} \times 1^{\prime}$ and a spectral resolution of 1750 at $4650 \AA$ to 3750 at $9300 \AA$. We used the Galaxy IFU Spectroscopy Tool (GIST $^{1}$ version 3.0.3; Bittner et al. 2019) pipeline to study the properties of UVIT J2022. GIST uses a python-implemented version of GANDALF (Sarzi et al. 2006; Falcón-Barroso et al. 2006; Bittner et al. 2019) and penalized pixel-fitting (pPXF; Cappellari \& Emsellem 2004; Cappellari 2017) to provide emission-line properties and stellar kinematics, respectively. We Voronoi-binned the data based on $\mathrm{H} \alpha(6558-6568 \AA)$ emission. We used a signal-to-noise ratio $(\mathrm{S} / \mathrm{N})$ of 30 for binning the data. We fitted the continuum using a multiplicative eighth-order Legendre polynomial. We removed noisy data that had a $\mathrm{S} / \mathrm{N}$ of less than 5 before binning the data.

\section{Results}

The left panel of Fig. 1 shows the DECaLS grz colour image for NGC 6902A, which is classified as an interacting galaxy (de Vaucouleurs et al. 1991a). The south-west outer region of

1 https://abittner.gitlab.io/thegistpipeline/ 

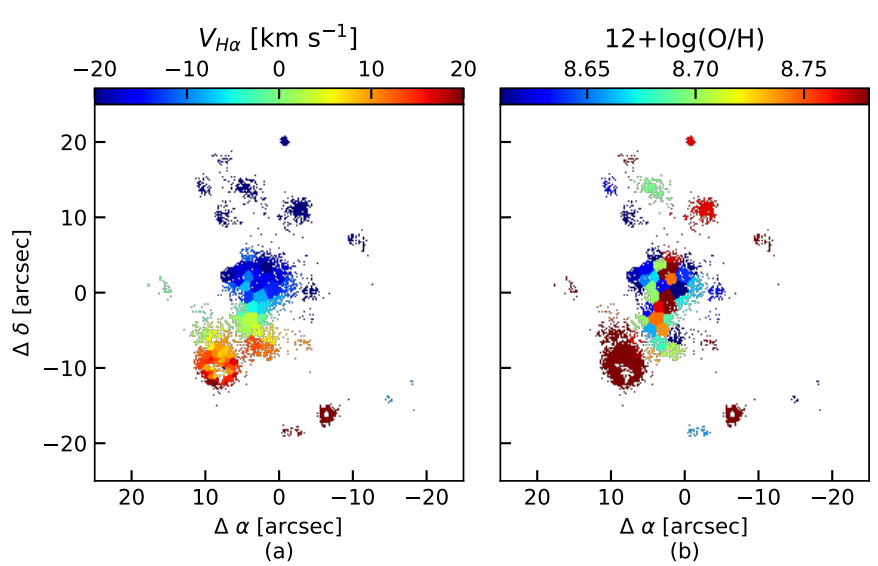

Fig. 2. Velocity and metallicity map of UVIT J2022. Panels $a$ and $b$ : $\mathrm{H} \alpha$ velocity map and metallicity map, respectively. The maps show the bins with an amplitude-to-noise ratio of more than 4 in the $\mathrm{H} \beta$, [O III] $\mathrm{H} \alpha$, and $[\mathrm{N} \mathrm{II}]$ lines. The origin $(0,0)$ in the maps are the coordinates of Table 1.

NGC 6902A in the DECaLS colour image shows diffuse blue emission. This south-western region shows prominent SFCs in the FUV image and corresponds to strong $\mathrm{H} \alpha$ emission from the MUSE cube, as shown in the right panel of Fig. 1. NGC 6902A is bright in the $K$ band image, and the disk appears extended in the south-west. However, there is only faint FUV emission and almost no $\mathrm{H} \alpha$ emission from NGC 6902A at the rest-frame wavelength.

This prompted us to investigate the peculiar feature in more detail to determine the cause of the interactions and to understand if this feature is the remnant of another galaxy that went through a merger with NGC6902A. To do so, we used $\mathrm{H} \beta$, [O III], $\mathrm{H} \alpha$, and [N II] lines of various SFCs in this region for redshift estimation. The mean redshift calculated from the emission lines for these SFCs is $z=0.00980 \pm 0.00018$, whereas the redshift of NGC 6902A is $z=0.05554$ (da Costa et al. 1991). This means that the diffuse blue emission was, in fact, from a foreground galaxy, which we discovered using FUV, MUSE, and $K$ band data. We have named it UVIT J202258.73-441623.8 (hereafter referred to as UVIT J2022). In the following sections, we described the detailed study we carried out to establish the characteristics and morphology of UVIT J2022.

\subsection{Neutral gas content, gas kinematics, and metallicity}

To determine the gas content of UVIT J2022, we looked for neutral gas line emission (HI) in the HI Parkes All Sky Survey (HIPASS) database using the location of UVIT J2022 and the appropriate redshift. We found some weak emission associated with the galaxy at an approximate velocity of $v_{\text {sys }}=2930 \mathrm{~km} \mathrm{~s}^{-1}$. The flux value is $\sim 0.032 \mathrm{Jy}$ beam $^{-1}$. Since the HIPASS beam is quite large $\left(\sim 15.5^{\prime}\right)$ and the diameter of UVIT J2022 is $\sim 1.5^{\prime}$, we can safely assume that all the HI emission lies within the beam. The neutral gas mass, including the contribution from $\mathrm{HI}$ gas and helium, is given by $M=1.4 \times 2.36 \times 10^{5} \times D_{\mathrm{Mpc}}^{2} \times$ $S_{v} \Delta V=1.85 \times 10^{9} M_{\odot}$, where $D_{\mathrm{Mpc}}$ is the distance in Mpc to UVIT J2022, $S_{v}$ is the flux in Jy, and $\Delta V$ is the width of the line, $\sim 100 \mathrm{~km} \mathrm{~s}^{-1}$. Excluding the contribution of helium, the HI gas mass is $M(\mathrm{HI})=1.32 \times 10^{9} M_{\odot}$.

UVIT J2022 is much brighter in $\mathrm{H} \alpha$ than the old stellar disk, which is faint and diffuse in the IRSF $K$ band image. We used
Table 1. Details of UVIT J2022.

\begin{tabular}{lc}
\hline \hline Source & UVIT J202258.73-441623.8 \\
\hline RA $(\mathrm{J} 2000)$ & $20: 22: 58.73$ \\
Dec $(\mathrm{J} 2000)$ & $-44: 16: 23.8$ \\
$z$ & $0.00980 \pm 0.00018$ \\
Distance & $\sim 41.86 \mathrm{Mpc}$ \\
$V_{\text {sys }}$ & $\sim 2930 \mathrm{~km} \mathrm{~s}$ \\
Inner disk radius & $\sim 4 \mathrm{kpc}$ \\
Outer disk radius & $\sim 9 \mathrm{kpc}$ \\
HI mass & $1.32 \times 10^{9} M_{\odot}$ \\
Stellar mass & $8.72 \times 10^{8} M_{\odot}$ \\
$\log \left(\Sigma_{\text {SFR }}\left(M_{\odot} \mathrm{yr}^{-1} \mathrm{kpc}^{-2}\right)\right) \mathrm{FUV}$ & -0.74 \\
$\log \left(\Sigma_{\mathrm{SFR}}\left(M_{\odot} \mathrm{yr}^{-1} \mathrm{kpc}^{-2}\right)\right) \mathrm{H} \alpha$ & -0.68 \\
\hline
\end{tabular}

Notes. The HI mass excludes the contribution of helium.

$\mathrm{H} \alpha$ to derive the velocity field of this galaxy. Figure 2a shows the $\mathrm{H} \alpha$ velocity map of the galaxy. It indicates galactic rotation with a velocity range of $\sim-20$ to $20 \mathrm{~km} \mathrm{~s}^{-1}$. The smaller range in velocity could be due to the face-on orientation of the galaxy.

Figure $2 \mathrm{~b}$ shows the metallicity map of UVIT J2022 derived using the following equation from Pettini \& Pagel (2004):

$12+\log (\mathrm{O} / \mathrm{H})=8.73-0.32 \times \log [([\mathrm{O} \mathrm{III}] / \mathrm{H} \beta) /([\mathrm{N} \mathrm{II}] / \mathrm{H} \alpha)]$.

The metallicity map shows that the galaxy has a higher metallicity along the north-west to south-east direction, which could be due to a small bar-like feature in the stellar disk of UVIT J2022. The SFCs at the edges of the bar also show higher metallicity. Bars are known to funnel gas from the outer to the inner disks, leading to star formation and thus enhancing the inner disk metallicities of galaxies (Combes et al. 2014). The $F 148 \mathrm{~W}$ and $\mathrm{H} \alpha$ images also show bright SFCs along and at the edges of the bar.

\subsection{Decomposition using GALFIT}

We used GALFIT (Peng et al. 2002) to perform the 2D decomposition of NGC 6902A and UVIT J2022 in the $r$ band. Although we did have IRSF $K$ band data, the diffuse nature of the stellar disk made it difficult to quantify the disk properties with good $\mathrm{S} / \mathrm{N}$; hence, we used the $r$ band image instead. A good fit was obtained by fitting three Sersic functions to NGC 6902A and two Sersic functions to the foreground galaxy UVIT J2022. The best fit was used to obtain models for both galaxies, and they are shown in Fig. 3. We found that the effective radius, $r_{\mathrm{e}}$, of the first Sersic profile in the $r$ band is $7.57 \mathrm{kpc}$, which probably corresponds to a large diffuse disk. The effective radius for the second Sersic profile in the $r$ band is $2.35 \mathrm{kpc}$, corresponding to a central stellar bar. However, the bar is not visible in the $K$ band image, which may be due to its diffuse nature. The central disk brightness for the outer diffuse disk in the $r$ band is $\mu_{0}(r)=20.13 \mathrm{mag} \mathrm{arcsec}^{-2}$, after correcting for inclination and cosmological dimming. However, there may be contamination due to background galaxy NGC 6902A.

To understand the stellar disk in more detail, we used the surface brightness profile (i.e. the magnitude $\operatorname{arcsec}^{2}$ in the $z$ band) to derive the mass density. The mass-to-light ratio assumed for the $z$ band was $\mathrm{M} / \mathrm{L}=1.4$ (Du \& McGaugh 2020), which gives a total stellar mass of $M(*)=8.72 \times 10^{8} M_{\odot}$. Using this stellar mass $M(*)$ in the mass-metallicity relation (Tremonti et al. 2004), the metallicity of UVIT J2022 is $\sim 8.6 \pm 0.1 \mathrm{dex}$, which is 

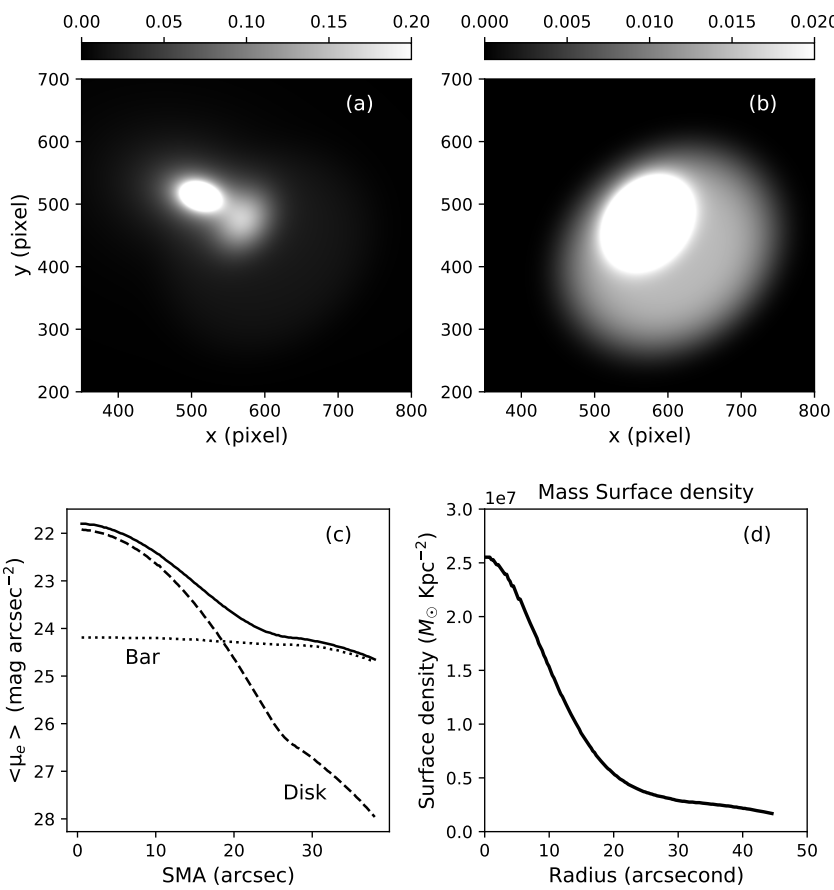

Fig. 3. GALFIT decomposition of NGC 6902A and UVIT J2022. Panel $a$ : model obtained from the fitting of NGC 6902A and UVIT J2022 with the $3+2$ Sersic component in the $r$ band. Panel $b$ : model image of UVIT J2022. The size scale is the same in panels a and b. However, the grayscale in panel $b$ is different to show the extent of UVIT J2022. Panel c: total model average surface brightness profile of UVIT J2022 in the $r$ band, obtained from GALFIT (solid curve). The dotted and dashed curves show the Sersic profiles for the inner central bar-like structure and disk component, respectively. Panel d: surface density of UVIT J2022.

consistent with the metallicity obtained from Eq. (1). The central region shows higher metallicity, possibly because of star formation triggered by the accumulation of gas funnelled from the outer disk into the inner region by the bar. Using the baryonic Tully-Fisher relation (McGaugh et al. 2000), the disk rotation velocity is $86_{-11}^{+14} \mathrm{~km} \mathrm{~s}^{-1}$. We found the inclination of UVIT J2022 to be around $13^{\circ}$ using

$V_{\mathrm{OBS}}=V_{\mathrm{ROT}} \times \sin (i)$,

where $V_{\mathrm{OBS}}$ and $V_{\mathrm{ROT}}$ are the observed and actual rotation velocity of the galaxy and $i$ represents the inclination of the galaxy.

The mass surface density is shown in panel d of Fig. 3 . The inner disk has a stellar mass surface density of $\Sigma(*) \approx 0.5$ to $2.5 \times 10^{7} M_{\odot} \mathrm{kpc}^{-2}$, whereas the outer disk has $\Sigma(*) \leq 4 \times$ $10^{6} M_{\odot} \mathrm{kpc}^{-2}$. The inner disk extends out to $\sim 20^{\prime \prime}$, or $4 \mathrm{kpc}$, and the outer disk extends out to $\sim 45^{\prime \prime}$, or $9.1 \mathrm{kpc}$. It is not surprising that only the inner disk shows star formation, as the outer disk is too diffuse and probably does not have enough disk gravity for gas to condense and form stars.

\subsection{Star formation history and star formation rate in UV and $H \alpha$}

Figure 4a shows the star formation history of the galaxy derived from GIST. The galaxy has gone through three bursts of star formation, separated by a few gigayears each. The latest episode of star formation is ongoing in this galaxy; this is evident from the $\mathrm{UV}$ and $\mathrm{H} \alpha$ images, which reveal the SFCs. We extracted the SFCs from the FUV $F 148 W$ and $\mathrm{H} \alpha$ images using the Python library for Source Extraction and Photometry (SEXTRACTOR;

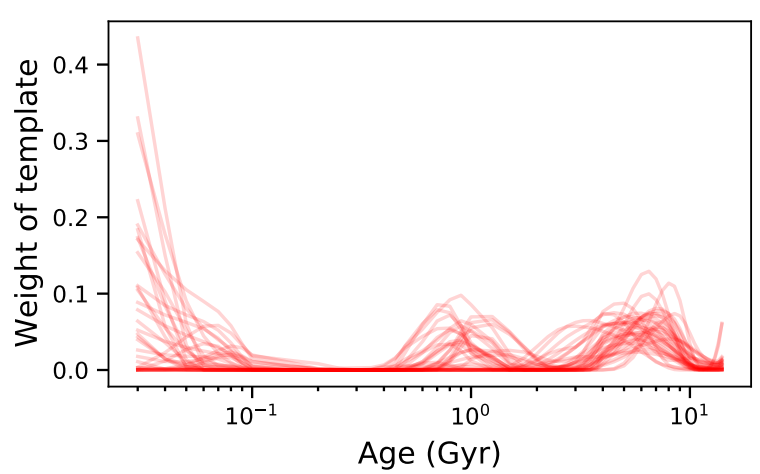

(a)

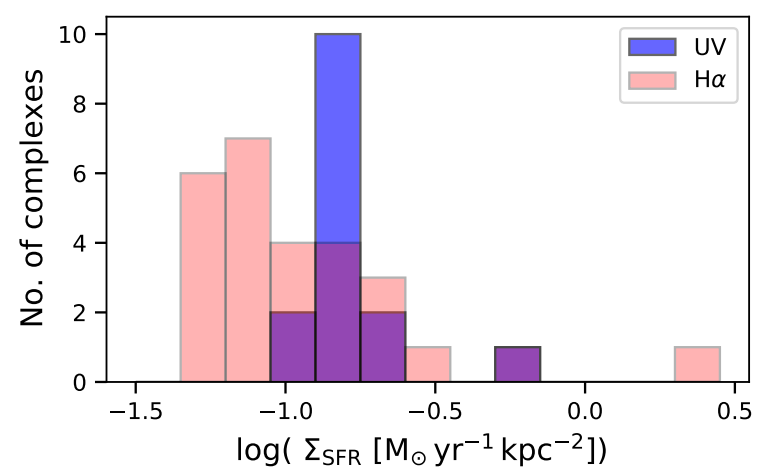

(b)

Fig. 4. SFH and SFR of UVIT J2022. Top panel: fraction of the stellar light that originates from a stellar population as a function of age. The curves represent individual bins from Sect. 2.2. Bottom panel: histograms of $\log \left(\Sigma_{\mathrm{SFR}}\right)$ of identified SFCs in UV and $\mathrm{H} \alpha$.

Bertin \& Arnouts 1996). We used a detection threshold of $5 \sigma$, where $\sigma$ is the global background noise. We detected 15 SFCs in the FUV and 27 SFCs in the $\mathrm{H} \alpha$, respectively. The number of detected FUV SFCs is lower because the FUV observations are not as deep as the $\mathrm{H} \alpha$ observations, so the fainter SFCs in the $\mathrm{H} \alpha$ may have been missed in the FUV. The spatial resolution of the MUSE $\mathrm{H} \alpha$ image is also higher than that of the UVIT FUV image.

We calculated the star formation rate per unit area $\left(\Sigma_{\mathrm{SFR}}\right)$ for each of the identified SFCs by performing elliptical aperture photometry on the identified sources using PHOTUTILS, a python ASTROPY package for photometry. We calculated the $\Sigma_{\text {SFR }}$ of each SFC from FUV emission using the method laid out in Salim et al. (2007, see Yadav et al. 2021a for more details) and $\mathrm{H} \alpha$ using that in Calzetti et al. (2007). Figures $4 \mathrm{a}$ and $\mathrm{b}$ show the star formation history and $\Sigma_{\mathrm{SFR}}$ for the FUV and $\mathrm{H} \alpha$ of UVIT J2022, respectively. The mean $\log \left(\Sigma_{\mathrm{SFR}}\left(M_{\odot} \mathrm{yr}^{-1} \mathrm{kpc}^{-2}\right)\right)$ estimated for UVIT J2022 from the FUV and H $\alpha$ are -0.74 and -0.68 , which is higher than in the XUV regions in galaxies as estimated by Thilker et al. (2007).

\section{Discussion}

We present the serendipitous discovery of a star-forming galaxy, UVIT J2022, at a redshift of $0.00980 \pm 0.00018$ $(D \approx 41.86 \mathrm{Mpc})$, which lies in the foreground of NGC 6902A $(z=0.05554 \pm 0.00010, D=253 \mathrm{Mpc}$; da Costa et al. 1991). The two had previously been classified as an interacting system (de Vaucouleurs et al. 1991a). However, the MUSE data clearly show that both galaxies are at different redshifts and are not interacting with each other. 
NGC 6902A shows signatures of a small perturbation of old stars towards the south-west region in the $K$ band. A few galaxies in the field have similar photometric redshifts, so the perturbed region could be due to a past dry merger. NGC 6902A is morphologically classified as SB(s)m pec in the de Vaucouleurs et al. (1991b) catalogue, and one of the Sersic functions used in our GALFIT decomposition represents a bar, thus confirming the de Vaucouleurs et al. (1991b) catalogue morphology.

Our detection of UVIT J2022 shows that diffuse star-forming galaxies can be missed in surveys but can be detected in UV and $\mathrm{H} \alpha$ observations, so the latter are very important for discovering diffuse LSBGs or UDGs at low redshifts. This is similar to the discovery of XUV disks via UV observations (Thilker et al. 2007). Also, spectroscopic observations are very important for avoiding projection bias and for detecting new galaxies (Yadav et al. 2021b).

The star formation history (Fig. 4) of UVIT J2022 shows that the galaxy has gone through two previous bursts of star formation, and the ongoing third burst is very prominent in the UV and $\mathrm{H} \alpha$. The bottom panel of Fig. 4 shows that the star formation rate of the extracted SFCs has similar mean values for FUV and $\mathrm{H} \alpha$ emission. UVIT J2022 also has spiral arms and a bar. Figure 2 shows that the spiral arm and bar in UVIT J2022 have enhanced metallicity. There is also higher metallicity in the outer disk than in the inner disk. The metal-enriched gas will increase gas cooling and can lead to further star formation in the central region (Martel et al. 2013), contributing to the buildup of the inner disk. The star formation history (Fig. 4) shows that UVIT J2022 is currently in the phase of rising star formation, so we expect the galaxy to become more luminous. Thus, UVIT J2022 is an example of a diffuse LSBG transforming into a luminous galaxy.

The central disk brightness for the outer diffuse disk in the $r$ band is close to the LSBG cutoff of $\mu_{0}(r)=21 \mathrm{mag} \mathrm{arcsec}^{-2}$ (Brown et al. 2001). Hence, although the inner disk is starforming, the outer disk is diffuse and similar to an LSBG in nature. The effective radius of UVIT J2022 in the $r$ band is $7.57 \mathrm{kpc}$ with a Sersic index of 0.52 , which is shallower than exponential decline $(n=1)$ and similar to spiral galaxies. The $\Sigma(*)$ of the inner disk is similar to that of the outer disks of XUV galaxies (Das et al. 2020), whereas the $\Sigma(*)$ of the outer disk is similar to that of dwarf LSBGs. The central surface brightness of UVIT J2022 is close to the limiting value for LSBGs (see Sect. 3.2), which are known to be dark-matter-dominated galaxies (de Blok \& McGaugh 1997). However, determining the dark matter mass of UVIT J2022 is difficult because the galaxy is close to face-on. Therefore, the HI line width or $\mathrm{H} \alpha$ velocity field will not give an accurate estimate of the disk rotation velocity. Future deep observations of the HI distribution can perhaps reveal something about the dark matter content. Thus, we conclude that UVIT J2022 is a diffuse, gas-rich LSBG in the process of disk growth via star formation in its inner disk. Its detection raises the possibility of finding similar diffuse systems at low redshifts using star formation tracers such as UV and $\mathrm{H} \alpha$. It also raises the intriguing question of how star formation is supported in such diffuse disks and whether disk dark matter may be playing a role (Das et al. 2020).

\section{Conclusions}

This Letter presents the serendipitous discovery of a starforming galaxy, UVIT J2022, at a redshift of $0.00980 \pm 0.00018$ lying in the foreground of NGC 6902A, which had mistakenly been classified as an interacting galaxy. Based on a detailed study using UVIT, DECaLS, and MUSE data, the following conclusions are inferred:

1. UVIT J2022 shows ongoing star formation in the UV and $\mathrm{H} \alpha$. It also shows a spiral arm and a bar-like feature.

2. The mean $\log \left(\Sigma_{\mathrm{SFR}}\left(M_{\odot} \mathrm{yr}^{-1} \mathrm{kpc}^{-2}\right)\right)$ of UVIT J2022 in the FUV and $\mathrm{H} \alpha$ is -0.74 and -0.68 , respectively.

3. UVIT J2022 has gone through three starburst phases, and the latest episode of star formation is ongoing.

4. The large outer, diffuse disk is low in surface brightness and has a low stellar density. So this galaxy is an example of a galaxy with an extended outer low surface brightness disk that is transforming into a luminous galaxy.

The discovery of a new foreground galaxy that was mistaken as a tidal feature of a bright background galaxy using powerful instruments such as UVIT and MUSE thus opens a gateway to searching for similar cases, where blue diffuse tidal features in interacting galaxies may not be the remnant of a merger but instead a separate foreground and/or background galaxy. It also shows the power of using star formation tracers such as FUV and $\mathrm{H} \alpha$ emission to detect diffuse galaxies.

Acknowledgements. We thank the anonymous referee for the thoughtful review, which improved the impact and clarity of this work. This Letter has used the data from the UVIT, which is part of the AstroSat mission of the Indian Space Research Organization (ISRO), archived at the Indian Space Science Data Center (ISSDC). This publication has also used near-infrared data from IRSF at SAAO. This Letter has also used the observations collected at the European Southern Observatory under ESO programme 0103.A-0637, run B. This research has also used data from DECaLS at CTIO. This publication has used the NASA/IPAC Extragalactic Database (NED), which is operated by the Jet Propulsion Laboratory, California Institute of Technology, under contract with the National Aeronautics and Space Administration. M.D. acknowledges the support of the Science and Engineering Research Board (SERB) MATRICS grant MTR/2020/000266 for this research.

\section{References}

Bacon, R., Accardo, M., Adjali, L., et al. 2010, in Ground-based and Airborne Instrumentation for Astronomy III, eds. I. S. McLean, S. K. Ramsay, \& H. Takami (SPIE), Proc. SPIE, 7735, 773508

Bertin, E., \& Arnouts, S. 1996, A\&AS, 117, 393

Bittner, A., Falcón-Barroso, J., Nedelchev, B., et al. 2019, A\&A, 628, A117

Brown, W. R., Geller, M. J., Fabricant, D. G., \& Kurtz, M. J. 2001, AJ, 122, 714 Calzetti, D., Kennicutt, R. C., Engelbracht, C. W., et al. 2007, ApJ, 666, 870 Cappellari, M. 2017, MNRAS, 466, 798

Cappellari, M., \& Emsellem, E. 2004, PASP, 116, 138

Chowdhury, A. 2019, MNRAS, 482, L99

Combes, F., García-Burillo, S., Casasola, V., et al. 2014, A\&A, 565, A97 da Costa, L. N., Pellegrini, P. S., Davis, M., et al. 1991, ApJS, 75, 935 Das, M., McGaugh, S. S., Ianjamasimanana, R., Schombert, J., \& Dwarakanath, K. S. 2020, ApJ, 889, 10

Das, M., Yadav, J., Patra, N., et al. 2021, JApA, 42, 85

de Blok, W. J. G., \& McGaugh, S. S. 1997, MNRAS, 290, 533

de Vaucouleurs, G., de Vaucouleurs, A., Corwin, H. G., Jr., et al. 1991a, Third Reference Catalogue of Bright Galaxies

de Vaucouleurs, G., de Vaucouleurs, A., Corwin, H. G., Jr., et al. 1991b, Sky and Telesc., 82, 621

Du, W., \& McGaugh, S. S. 2020, AJ, 160, 122

Falcón-Barroso, J., Bacon, R., Bureau, M., et al. 2006, MNRAS, 369, 529

Fattahi, A., Navarro, J. F., \& Frenk, C. S. 2020, MNRAS, 493, 2596

Flaugher, B., Diehl, H. T., Honscheid, K., et al. 2015, AJ, 150, 150

Honey, M., van Driel, W., Das, M., \& Martin, J. M. 2018, MNRAS, 476, 4488

Impey, C., \& Bothun, G. 1997, ARA\&A, 35, 267

Koda, J., Yagi, M., Yamanoi, H., \& Komiyama, Y. 2015, ApJ, 807, L2

Kumar, A., Ghosh, S. K., Hutchings, J., et al. 2012, in Space Telescopes and Instrumentation 2012: Ultraviolet to Gamma Ray, eds. T. Takahashi, S. S. Murray, \& J. W. A. den Herder, SPIE Conf. Ser., 8443, 84431N

Leisman, L., Haynes, M. P., Janowiecki, S., et al. 2017, ApJ, 842, 133

Lim, S., Côté, P., Peng, E. W., et al. 2020, ApJ, 899, 69

Martel, H., Kawata, D., \& Ellison, S. L. 2013, MNRAS, 431, 2560

McGaugh, S. S., Schombert, J. M., Bothun, G. D., \& de Blok, W. J. G. 2000, ApJ, 533, L99 
A\&A 657, L10 (2022)

Menci, N., Fontana, A., Giallongo, E., \& Salimbeni, S. 2005, ApJ, 632, 49

Nagayama, T. 2012, African Skies, 16, 98

Nagayama, T., Nagashima, C., Nakajima, Y., et al. 2003, in Instrument Design and Performance for Optical/Infrared Ground-based Telescopes, eds. M. Iye, \& A. F. M. Moorwood, SPIE Conf. Ser., 4841, 459

Peng, C. Y., Ho, L. C., Impey, C. D., \& Rix, H.-W. 2002, AJ, 124, 266

Pettini, M., \& Pagel, B. E. J. 2004, MNRAS, 348, L59

Postma, J. E., \& Leahy, D. 2017, PASP, 129, 115002

Postma, J. E., \& Leahy, D. 2020, PASP, 132, 054503

Prole, D. J., van der Burg, R. F. J., Hilker, M., \& Davies, J. I. 2019, MNRAS, 488, 2143

Rong, Y., Zhu, K., Johnston, E. J., et al. 2020, ApJ, 899, L12
Sales, L. V., Navarro, J. F., Peñafiel, L., et al. 2020, MNRAS, 494, 1848 Salim, S., Rich, R. M., Charlot, S., et al. 2007, ApJS, 173, 267

Sancisi, R., Fraternali, F., Oosterloo, T., \& van der Hulst, T. 2008, A\&ARv, 15, 189

Sarzi, M., Falcón-Barroso, J., Davies, R. L., et al. 2006, MNRAS, 366, 1151 Somerville, R. S., Primack, J. R., \& Faber, S. M. 2001, MNRAS, 320, 504 Thilker, D. A., Bianchi, L., Meurer, G., et al. 2007, ApJS, 173, 538

Tremonti, C. A., Heckman, T. M., Kauffmann, G., et al. 2004, ApJ, 613, 898 van Dokkum, P. G., Abraham, R., Merritt, A., et al. 2015, ApJ, 798, L45 Venhola, A., Peletier, R., Laurikainen, E., et al. 2018, A\&A, 620, A165 Yadav, J., Das, M., Patra, N. N., et al. 2021a, ApJ, 914, 54

Yadav, J., Das, M., Barway, S., \& Combes, F. 2021b, A\&A, 651, L9 